\title{
A Summary of Optimal Methods for the Planning of Stand-alone Microgrid System
}

\author{
Lei Qiao \\ School of Control and Mechanical Engineering, Tianjin Institute of Urban Construction, Tianjin, China \\ Email: qiaolei@tjuci.edu.cn
}

Received February, 2013

\begin{abstract}
This paper describes the characteristics and optimal methods for the planning of stand-alone microgrid system, in order to improve the power supply reliability, increase the coefficient of utilization of renewable energy and reduce the cost of investment and operation. Next, the problems in the optimal planning for a stand-alone microgrid system are summarized, including the unique operational control targets, the flexible combination approaches and the operation strategies of distributed generation energy supply system, and the special requirements of the reliability of power supply quality factor from the different users. And then, centering on the operational control and the advanced energy management strategy, the optimal mathematical models and the solving methods, the reliability assessment approaches and the improvement measures of a stand-alone microgrid system, an overview of the general situation of the recent research at home and abroad and the limitations of the study are summarized. Finally, several problems, existing in the optimal planning of stand-alone microgrid system, to be urgently solved, are put forward.
\end{abstract}

Keywords: Stand-alone Microgrid System; Optimal Planning; Operation Strategies; Energy Management

\section{Introduction}

At present, considering geographical conditions, it is rather difficult to build a conventional power distribution system that connects with the power grid in some remote areas and stand-alone islands. Diesel engine is usually adopted as the main electrical source, nevertheless the supply mode has many shortcomings, such as the low reliability, the high operation and maintenance cost and the environmental pollution, and so on. A stand-alone microgrid system integrates the conventional power generation technology, the distributed generation technology and the storage energy devices by a reasonable electric network, and is the most effective way of improving the supply reliability, increasing the utilization rate of renewable energy resources, saving the operation expense, lowering the energy consumption, reducing the pollutants discharge, and realizing the optimal use of multiple energy resources. Accordingly, the power energy demands of remote areas and stand-alone islands can be met better through a stand-alone microgrid.

Presently, some demonstration projects of stand-alone microgid have already been built all over the world, such as the microgid system with wind turbines, photovoltaic arrays, diesel engines and energy storage devices on Kythnos island in Greece [1] and the stand-alone microgid consisting of multiple energy forms and seawater desalination installations on Dangan island in Zhuhai [2].
The above-mentioned projects only explored the question about power supply to island from a feasibility viewpoint, but the genuine optimal planning has been not still achieved as a result of deficiencies in the theory and method of planning.

According to the character of optimal planning for a stand-alone microgrid system, the article analyzes the research status of optimal methods for the planning of stand-alone microgrid system, summarizes the research limitations, and at last proposes the pivotal problems needed to be solved urgently.

\section{A Brief Description of Optimal Planning for a Stand-alone Microgrid System}

\subsection{Planning Objectives}

The main planning objective for a stand-alone microgrid is to decide the optimal system scheme that makes the expense of construction and operation lowest on the basis of the power energy demand, the renewable energy supply and the condition of existing power network in the planning period.

Figure 1 shows a diagram of a simple radial $10 \mathrm{kV}$ AC stand-alone microgrid system, where DG (distributed generator)contain diesel generators, photovoltaic system, wind turbine generators and fuel cells, and ESS represents the energy storage system, they are connected to 


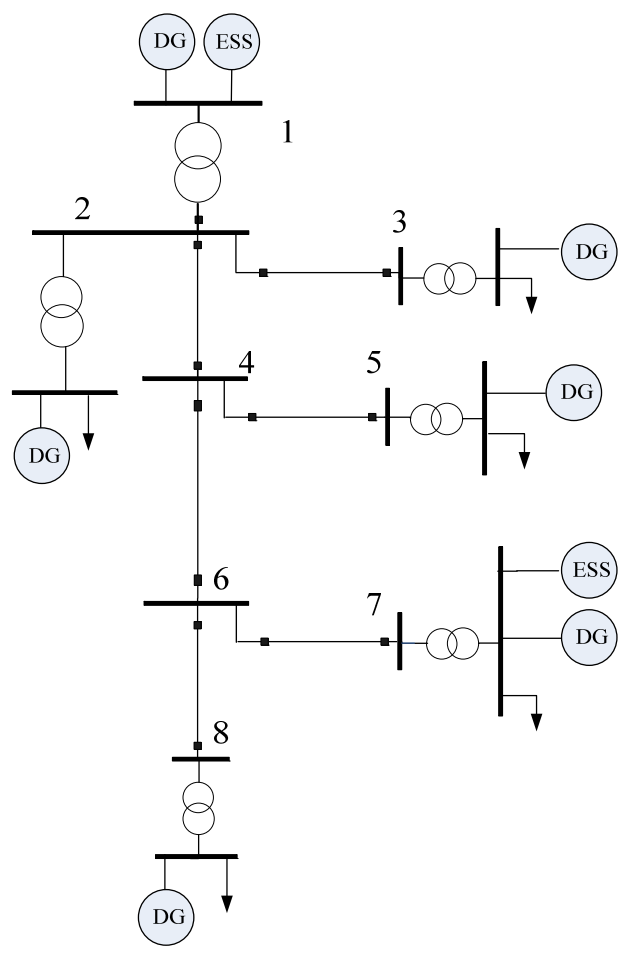

Figure 1. AC topology of a stand-alone microgrid system.

low voltage $\mathrm{AC}$ buses dispersedly and then input the $10 \mathrm{kV}$ or more high voltage grid through booster transformers. Based on the structure, the main research contents of the planning for a stand-alone microgrid include the planning of capacity of distributed generation and energy storage devices and the network frame planning. The former carries out the optimal design to choose the type, capacity and control strategy of distributed generators in a microgrid from the viewpoint of the balance between the supply and demand, and the latter studies the optimal planning about the structure of power network, the optimal path and the connected location of distributed generation. In the areas with grids, the key of the planning is how to choose reasonably the capacity and location of the energy supply system with distributed generators to satisfy the established objectives, on the condition that the comprehensive energy demand is met, according to the situation of local resources. Whereas, in some areas that need the overall transformation or is non-electric, the joint optimal planning should be developed.

\subsection{Character of Optimal Planning}

In a stand-alone microgrid system, many factors make modeling and solving of the optimal planning very complex, such as the input of intermittent renewable energy resources, the flexible combination approaches, the various control strategies and the different demand for the power supply reliability. There are the main representa- tions.

1) There are a mass of uncertain factors in a standalone microgrid system, and hence the more flexible model and solution algorithm of optimal planning need to be adopted.

The planning period of stand-alone microgrid system is usually $10-20$ years. The planning scheme is established based on the long period forecast of the comprehensive energy demand and some renewable energy resources. Nevertheless, the result of forecast is uncertain as a result of external conditions, such as the weather. In addition, the fluctuation of the price of fossil energy and the decreasing investment cost of distributed generators also produce an effect on the optimal planning scheme.

2) A stand-alone microgrid system has the particular operation and control objectives, the flexible combination schemes and the control strategies that influence the result of the optimal planning, consequently these factors should be considered adequately.

A microgrid system that is able to connect grid links the power system in most of the time. The optimal objectives of the maximal incomes and the minimal pollutant emission can be achieved by managing scientifically the different units in a microgrid system [3,4]. A stand-alone microgrid system is not supported by the power system, accordingly its main operational objective is to maintain the long-term stability of system and meet the demand of power energy. In a stand-alone microgrid system, the combination schemes and the operational modes of distributed generators and energy storage devices are more flexible, and as for the control strategies, the methods of coordination control are multiform [5].

3) When a stand-alone microgrid system is programmed, the different users' demands for the power supply reliability should be considered, and then according to the evaluation result of reliability, the planning scheme needs to be modified.

A stand-alone microgrid system contains the multiple energy input, the uncertainty of optimal planning, the various combination schemes of distributed units, the flexible system structure and the different demands for the power supply reliability, which undoubtedly increase the difficulty of the modeling and solution method of the optimal planning.

\section{Research Status of Optimal Planning for Stand-Alone Microgrid System}

The research status of the optimal planning for a standalone microgrid system is introduced, including the operational control and the energy management strategies, the mathematical model and solution algorithm of optimal planning, and the evaluation methods of reliability, etc. 


\subsection{Operational Control and Energy Management Strategy of Stand-alone Microgrid System}

The existing researches demonstrate that a stand-alone microgrid system with multiple energy systems can increase the efficiency and the energy utilization factor. However, due to the particular operational modes and the various combination schemes of system, the feasible control strategy need to be used in order to ensure the system stability.

At present, the controls of microgrid are classified into the control of distributed generators and the energy management.

1) Operational strategies

The controls of distributed generators are divided into constant power control, droop control and constant voltage /constant frequency control. The control strategies of microgrid are classified into master-slave control and equivalence control. Up till now, in the existing standalone microgrid system, the layered management mode based on master-slave control is adopted frequently. In the control strategy, the adjustable generators such as diesel generators, gas turbines and biomass generation power, or the energy storage devices are disposed as the main units of voltage regulation and frequent regulation, besides other distributed generators are controlled with a constant power.

The adjustable generators in a microgrid adopt the plug and play equivalence control to realize the equipartition of active current and reactive current without the real-time communication, accordingly the reliability of power supply is improved.

When a synchronous generator acts as distributed generation, the equivalence control is realized easily as a result of the inherent droop character of synchronous generator. Besides, some experts proposed many measures to achieve the equivalence control of inventor, and the chief method was to simulate the droop control of the regulation character of synchronous generator [6-8]. The main disadvantage of droop control is that the frequency and the voltage both have a steady state error. Consequently, the energy storage devices were used in the second frequency regulation in [9] and a dynamic voltage recovery equipment was introduced in order to reduce the voltage tolerance in [10]. The results of reference [11] showed that the energy storage devices could ensure the voltage stability and frequency stability of isolated system by controlling the power appropriately in a standalone microgrid where synchronous generators acted as the main electrical source. For a stand-alone microgrid, the deviation of frequency and voltage can be improved by using the upper management system to dispatch the power generation and supply.
2) Advanced energy management strategy

All kinds of distributed units in a system are independent relatively and yet coupled, so the coordinated relationship between energy resources and energy consumption devices should be considered to realize the comprehensive utilization of multiple energy resources.

Reference [12] suggested several operational strategies around the energy management of diesel generator and energy storage battery - the strategies decided the operational priority of diesel generator and energy storage battery based on the comparison the unit generation cost of diesel generator with one cycle charging and discharging cost of energy storage battery, the method was applied in a optimal software-HOMER. The energy management strategy of stand-alone microgrid with fuel cells, electrolytic water equipments and electrochemical cells was researched, and a approach to coordinating and dispatching the hybrid energy storage was proposed based on the battery's state of charge in [13]. Considering the constraint condition of the equipment operation, the coordinated control strategies of multiple energy resources were suggested to ensure the long-term and reliable operation of energy storage batteries in [14]. The achievements above indicated how to select a combination scheme and control strategies of distributed generation system depended on the local renewable energy resources, the load demands and the cost of equipments and fuel. In addition, at present some simulation softwares can supply the coordination control strategies of distributed generators. For example, simulation software Hybrid2 developed by NERL suggests several schemes that are divided into two classes [5], one is that diesel generators play a role of net load following and storage batteries are in the condition of floating charge as the reserved power, and the other is that diesel generators and storage batteries serve as the main power supply in turn to meet the demand of net load.

\subsection{Mathematics Model and Solution Methods of Optimal Planning for Stand-alone Microgrid System}

Presently, in the aspect of optimal planning for a standalone microgrid, many scholars at home and abroad mainly focus on designing the capability of distributed generators from the viewpoint of balance of supply and demand. The most of researches calculate every index of the combination scheme of different renewable energy resources with the quasi steady state simulation program according to the load data, the wind speed, the illumination intensity and the temperature in a life cycle - the method is called the deterministic method. The advantage of means is that the fluctuation of renewable energy resources and loads in a life cycle and control strategies 
are simulated in detail during the optimal planning. But, the disadvantage is that the forecast errors and the price fluctuation influence the result of optimal planning, and the uncertain factors can not be estimated. In addition, the hourly data of weather and load need to be known in the process of planning, however, it is difficult in practice.

Accordingly, the uncertain theory is applied widely in the generators expansion planning and the system planning. The uncertain planning aims at the optimal problems in the uncertain conditions, and explains the integrated theory of modeling and solving of stochastic planning, fuzzy planning and rough planning [15]. Reference [16] researched the planning of distribution network containing distributed generators with the fuzzy optimal method. Reference [17] used Monte Carlo simulation to simulate illumination radiant intensity and the initial capability of batteries and adopted the quasi steady state simulation to analyze the reliability index during the optimal design, and then the result with meeting a certain confidence level was regarded as the convergence criterion of Monte Carlo simulation. Reference [18] advanced the optimal configuration model of wind-hydro-solar generation system based on the stochastic chance-constrained programming to obtain the optimal configuration schemes and the evaluation indexes with meeting the objectives and constraint conditions in all kinds of confidence levels.

The optimal objectives involve the reliability, the system cost and the pollutant emission. The reliability indexes contain loss of load probability, loss of load probability and loss of load hours, and so on [19]. The costs of system include the net cost and standardization cost in a life cycle [20-22]. The problem is the hybrid optimal planning with discrete variables and continuous variables, so the artificial intelligent algorithm is fit to solve the problem [23]. Reference [24-26] designed the type and capability of distributed generators with the genetic algorithm. Multiple constraint conditions exist at the same time during the optimal program, but in the practical multi-objectives optimal problem, the different objectives always conflict one another. The multi-objectives optimal design was achieved based on a fixed weigh in [27] that converted the multi-objectives to the single objective. But, the multi-objectives solving does not mean looking for the single optimal solution but searching a set of equilibrium solution- Pareto optimal solution. The multi-objectives genetic algorithm was adopted to design the capability of stand-alone wind-solar-diesel-battery microgrid, and aimed at the lowest cost of life cycle and the minimum of carbon emission in [28]. Reference [29] proposed a three-objective model containing the probability index of capacity deficiency. Reference $[24,30]$ described a layered optimal design, outer layer calculated the capability of distributed generators, and the internal layer optimized the key control variables of the selected control strategy, and the method was applied in an optimal software-HOGA.

Besides the researches mentioned, the distributed generation technology brings a new challenge to distribution systems [31-32], accordingly, the comprehensive coordination between distributed generators and distribution systems should be considered adequately while the optimal planning is laid out.

Reference [33] pointed out the planning of distribution system with distributed generators was divided into the location planning of distributed generators and the expanding planning of distribution systems. Reference [31] built a planning model of distribution grid with distributed generators considering the security restriction, the randomness of distributed generator's output, the power penetration of distributed generators and the joint planning of distribution grids and distributed generators. Reference [34] used the chance constrained programming to set up a grid structure planning model for distribution networks with distributed wind turbine generators, considering the randomness of wind power generation and the uncertainty of load forecast.

As already mentioned, the optimization of capability, location and structure of distributed generation had already been carried out, however the optimal planning for a stand-alone microgrid was hardly reported.

\subsection{Reliability Evaluation and Improvement Measures for Stand-Alone Microgrid System}

The theory of reliability is applied in the planning in order to establish the reasonable strategy and look for the optimal balance between the economical efficiency and the reliability, on the premise of meeting the reliability evaluation index. For a stand-alone microgrid, the present researches mostly focus on the reliability evaluation of power output and load need, and the purpose is the quantization and analysis of risk as a result of the random invalidation of system, at the same time, not the index of single load point but the general adequacy index should be supplied. Reference [35] regarded the loss of load expectation (LOLE) and the loss of energy expectation (LOEE) as a reliability evaluation index, and discussed the solving of reliability for a stand-alone microgrid with wind turbine generators and batteries. Reference [36] studied the effect of control strategy and configuration scheme on the reliability index of stand-alone microgrid.

Another important aspect of reliability evaluation is that the correct measure should be adopted to adjust the output power of generators and loads during analyzing the state of system. From the viewpoint of the balance 
between output power and load demand, besides the best strategy of cutting load, the optimal strategy of switching of renewable energy resources and rectification of power need to be researched. According to the character of dispersed collocation and approaching load, when the arranged overhaul and the unexpected fault occurs, the system can be divided into several stand-alone sub-microgrids, meanwhile, the divided principle and reliability of sub-microgrid should be taken into account. Reference [37] built an isolated model of distribution network in the basis of the importance of loads, and aimed at the maximal equivalent effective load. Reference [38] researched a computational method of probability of forming isolated island. Reference [39] discussed the effect of isolated island operation on reliability. Reference [40] could obtain the feasible dividing scheme in shorter time with the heuristic search, according to the request of load balance in the isolated mode.

\subsection{Research Limitations}

Through analyzing the above-mentioned research status, we can see that there are some limitations around the theory and method of optimal planning for a stand-alone microgrid. The main representations are as follows:

1) In the aspect of mathematical model and solution methods of optimal planning

Up till now, the researches at home and abroad have not involved the joint planning of power source and power system, which was the key of taking full advantage of renewable energy resources. Multiple management strategies of optimal planning don't consider the integrated optimization of single unit capability and numbers of devices, the combined operation mode of devices, and the calculation of reserve capacity, and so on. The results gained only based on the energy balance in a simulation step are infeasible in some ways.

2) In the aspect of methods of optimal planning in the uncertain circumstance

Many works focus on the stochastic optimization based on the uncertainty of probability and ignore the effect of uncertain factors on the planning results. Besides, because the problem is the joint planning of distributed generators and power system, and included the selection of site and capability, the choice of control parameter and the system structure planning, if a single layered optimal planning is used, the problem of curse of dimensionality tends to occur.

3) In the aspect of reliability analysis and innovative approach for a stand-alone microgrid

In the research of reliability evaluation, the reliability is often analyzed from the viewpoint of the balance between supply and demand, and no attention is paid to the factors of the reliability of non- electric components, network structure and fault type. However, the reliability of system can be improved effectively by designing and installing reasonably the relay protection and automation devices, so the reliability evaluation of the full system with generators and electric components should be carried out.

In the aspect of improving the reliability of standalone microgrid, the division of stand-alone microgrid, the correction measure of load in the condition of fault, and the switch and adjustment strategy of power of renewable energy resources need to be researched in depth.

Compared with the developed countries, our researches focus on the optimal design of complementary power system structure, the control of rock-bottom device and the system simulation. Moreover, there is a lack of the theory and guidance of stand-alone microgrid and the corresponding optimal tools.

\section{Research Prospect}

The objective of optimal planning for a stand-alone microgrid is to look for the planning scheme of isolated system with distributed generators, and meet the operational constraints and the load reliability constraints. The optimal design needs to take account into the control strategy, so the operation and planning are coupled each other. Accordingly, the modeling and solving in the planning become more complex. Future researches should be developed as follows.

1) Research of multi-objective optimal planning for a stand-alone microgrid in uncertain circumstances

All kinds of energy resources are not only independent but also coupled. The emphases of research include the energy management strategies of stand-alone sub-microgrid, the modeling method considering uncertainty factors of consumer demand, the condition of renewable energy resources and market price, the mathematical model of the multi-objective optimal planning for a stand-alone microgrid with distributed generators and the solving method of uncertain planning theory.

2) Reliability evaluation and innovative approach for a stand-alone microgrid

The reliability of stand-alone microgrid is influenced by the type of fault, the system structure, the energy management strategy and the operational mode, consequently the optimal planning scheme needs to be evaluated to advance the innovative measures. When the reliability is analyzed, the intermittence of renewable energy resources, the uncertainty of load, the variable operation mode of energy storage system and the fault character of devices must be investigated. The corrective actions should be discussed, including the division method of sub-microgrids, the optimal correction strategy of loads in the condition of fault, the best switching way of re- 
newable energy resources generating equipment and the power adjusting measure of adjustable units.

3) Two layered uncertain planning method with the reliability for a stand-alone microgrid

In the condition that the total capability of system is known, and the load demand, the reliability index and the operational restraint are met, Researchers are confronted with a problem that is how to find a set of optimal decision variables results in the minimal total expense of investment, operation and loss and increasing the probability of joining up renewable energy resources. Therefore, the joint planning of power source and power system should be studied.

The joint optimal planning for a stand-alone microgrid contains not only the planning of system structure, but also the optimization of the location and capability of access of distributed generation. The solving of the problem is complex, and the multi-layered uncertain planning method is adopted to decrease the difficulty. Consequently, the modeling and solving of two layered uncertain planning should be developed.

\section{Conclusions}

This paper introduces the characteristics and problems of the planning of stand-alone microgrid system, and summarizes the general situation of the recent research at home and abroad and the limitations of the study. Finally, several problems, existing in the optimal planning of stand-alone microgrid system, to be urgently solved, are put forward.

\section{REFERENCES}

[1] M. Vandenbergh, R. Geipel, M. Landau and P. Strauss, "Performance Evaluation of the Gaidoroumandra Mini-grid with Distributed PV Generators," 4th European PV-Hybrid and Mini-Grid Conference, Athens, 29-30 ${ }^{\text {th }}$ May 2008.

[2] K. L. Wang, Y. G. You and Y. Q. Zhang, "Energy Management System of Renewable Stand-lone Energy Power Generation System in an Island," Automation of Electric Power Systems, Vol. 34, No. 14, 2010, pp. 13-17.

[3] C. Chen, S. Duan, T. Cai, B. Liu, and G. Hu, "Smart Energy Management System for Optimal Microgrid EcoNomic Operation," Renewable Power Generation, IET, Vol. 5, No. 3, 2011, pp. 258-267. doi:10.1049/iet-rpg.2010.0052

[4] X. P. Liu, M. Ding, Y. Y. Zhang and N. Z. Xu, "Dynamic EcoNomic Dispatch for Microgrids," Proceedings of the CSEE, Vol. 31, No. 31, 2011, pp. 77-84.

[5] J. F. Manwell, A. Rogers, G. Hayman, C. T. Avelar and J. G. McGowan, et al., "Hybrid2-a Hybrid System Simulation Model: Theory Manual," Renewable Energy Research Laboratory, University of Massachusetts, Massachusetts, 2006.
[6] R. H. Lasseter, "MicroGrids and Distributed Generation," Journal of Energy Engineering American Society of Civil Engineers, Vol. 133, No. 3, 2007, pp. 144-149. doi:10.1061/(ASCE)0733-9402(2007)133:3(144)

[7] C. S. Wang, Z. X. Xiao and S. X. Wang, "Multiple Feedback Loop Control Scheme for Inverters of the Micro Source in Microgrids," Transactions of China Electrotechnical Society, Vol. 24, No. 2, 2009, pp. 100-107.

[8] J. M. Guerrero, L. G. de Vicuńa, J. Matas, N. Berbel and J. Sosa, "Wireless-control Strategy for Parallel Operation of Distributed-generation Inverters," IEEE Transactions on Industrial Electronics, Vol. 54, No. 5, 2006, pp. 1461-1470 . doi:10.1109/TIE.2006.882015

[9] C. E. Jones, "Local Control of Micro Grids Using Energy Storage," Ph.D. Thesis, University of Manchester, Manchester, 2007.

[10] S. Morozumi and K. Nara, "Recent Trend of New Type Power Delivery System and its Demonstrative Project in Japan," IEEE Transactions on Power and Energy, Vol. 127, No. 7, 2007, pp. 770-775. doi: $10.1541 /$ ieejpes. 127.770

[11] L. Guo, C. S. Wang, "Dynamical Simulation on MicroGrid with Different Types of Distributed Generations," Automation of Electric Power Systems, Vol. 33, No. 2, 2009, pp. 82-86.

[12] C. D. Barley and C. B. Winn, "Optimal Dispatch Strategy in Remote Hybrid Power Systems," Solar Energy, Vol. 58, No. 4-6, 1996, pp. 165-179. doi:10.1016/S0038-092X(96)00087-4

[13] G. Giannakoudis, A. I. Papadopoulos, P. Seferlis and V. Spyros, "Optimum Design and Operation Under Uncertainty of Power Systems Using Renewable Energy Sources and Hydrogen Storage," International Journal of hydrogen energy, Vol. 35, No. 3, 2010, pp. 872-891. doi:10.1016/j.ijhydene.2009.11.044

[14] M. X. Liu, L. Guo and C. S. Wang, "Coordinated Operation Control Strategy for Stand-alone Wind-solar- diesel-battery Microgrid," Automation of Electric Power Systems, Vol. 36, No. 15, 2012, pp. 1-6.

[15] B. D. Liu, R. G. Zhao and G. Wang, "Planning and Application of Uncertainty Thoery," Tsinghua University Press, Beijing, 2003.

[16] Y. Yang, G. Wei, B. Zhou and X. Zhang, "Optimized Fuzzy Planning of the Distribution Network Including Distributed Generation," Automation of Electric Power Systems, Vol. 34, No. 13, 2010, pp. 19-23.

[17] P. Arun, R. Banerjee and S. Bandyopadhyay, "Optimum Sizing of PhotoVoltaic Battery Systems Incorporating Uncertainty through Design Space Approach," Solar Energy, Vol. 83, No. 7, 2009, pp. 1013-1025. doi:10.1016/j.solener.2009.01.003

[18] F. Zhang, Z. Cai and M. H. Yang, "Capacity Allocation of Rural Hybrid Generating System Based on Stochastic Chance Constrained Programming," Transactions of the Chinese Society of Agricultural Engineering, Vol. 26, No. 3, 2010, pp. 267-271.

[19] H. A. M. Maghraby, M. H. Shwehdi and G. K. Al-Bassam, "Probabilistic Assessment of PhotoVoltaic 
(PV) Generation Systems," IEEE Transactions on Power System, Vol. 17, No. 1, 2002, pp. 205-208. doi: $10.1109 / 59.982215$

[20] J. L. Bernal-Agustín, R. Dufo-López and D. M. Rivas-Ascaso, "Design of Isolated Hybrid Systems Minimizing Costs and Pollutant Emissions," Renewable Energy, Vol. 31, No. 14, 2006, pp. 2227-2244. doi:10.1016/j.renene.2005.11.002

[21] G. J. Dalton, D. A. Lockington and T. E. Baldock, "Feasibility Analysis of Stand-alone Renewable Energy Supply Options for a Large Hotel," Renewable energy, Vol. 33, No. 7, 2008, pp. 1475-1490. doi:10.1016/j.renene.2007.09.014

[22] M. L. Deshmuk and S. S. Deshmuk, "Modeling of Hybrid Renewable Energy Systems," Renewable and Sustainable Energy Reviews, Vol. 12, No. 1, 2008, pp. 235-249. doi:10.1016/j.rser.2006.07.011

[23] R. Baños, F. ManzaNo-Agugliaro, F. G. Montoya, C. Gil, and A. Alcayde, et al., "Optimizaiton Methods Applied to Renewable and Sustainable Energy: A Review," Renewable and Sustainable Energy Reviews, Vol. 15, No. 4, 2011, pp. 1753-1766.doi:10.1016/i.rser.2010.12.008

[24] R. Dufo-López and J. L. Bernal-Agustín, "Design and Control Strategies of PV-diesel Systems Using Genetic Algorithms," Solar Energy, Vol. 79, No. 1, 2005, pp. 33-46.doi:10.1016/i.solener.2004.10.004

[25] H. Yang, W. Zhou and C. Lou, "Optimal Design and TechNo-ecoNomic Analysis of a Hybrid Solar-wind Power Generation System," Applied Energy, Vol. 86, No. 2, 2009, pp. 163-169.doi:10.1016/j.apenergy.2008.03.008

[26] R. Carapellucci and L. GiordaNo, "Modeling and Optimization of an Energy Generation Island Based on Renewable TechNologies and Hydrogen Storage Systems," International Journal of Hydrogen Energy, Vol. 37, No. 3, 2011, pp. 2081-2093.

[27] H. Yang, W. Zhou, L. Lu and Z. H. Fang, "Optimal Sizing Method for Stand-alone Hybrid Solar-wind System with LPSP TechNology by Using Genetic Algorithm," Solar Energy, Vol. 82, No. 4, 2008, pp. 354-367. doi:10.1016/j.solener.2007.08.005

[28] R. Dufo-López, J. L. Bernal-Agustín, J. M. Yusta-Loyo, J. A. Dominguez-Navarro, and L. J. Ramirez-Rosado, et al., "Multi-objective Optimization Minimizing Cost and Life Cycle Emissions of Stand-alone PV-wind-diesel Systems with Batteries Storage," Applied Energy, Vol. 88, No. 11, 2011, pp. 4033-4041. doi:10.1016/j.apenergy.2011.04.019
[29] H. L. Song, J. Y. Wu, L. Y. Ji, L. Z. Gao, Y. L. Liu, et al. "Multi-Objective Optimal Sizing of Stand-alone Hybrid Wind/pV System," Transactions of China Electrotechnical Society, Vol. 26, No. 7, 2011, pp. 104-111.

[30] R. Dufo-López and J. L. Bernal-Agustín, "Optimization of Control Strategies for Stand-alone Renewable Energy Systems with Hydrogen Storage," Renewable Energy, Vol. 32, No. 7, 2007, pp. 1102-1126. doi:10.1016/j.renene.2006.04.013

[31] W. Ouyang, "Distribution Network Planning with Distributed," Ph.D. Thesis, Shanghai Jiao Tong University, Shanghai, 2009.

[32] J. C. Yu, F. J. Chi, K. Xu, S. W. Li and H. Liu, et al., "Analysis of the Impact of Distributed Generation on Power Grid," Proceedings of the CSU-EPSA, Vol. 24, No. 1, 2012, pp. 138-141.

[33] M. Wang and M. Ding, "Distribution Network Planning Including Distributed Generation," Proceedings of the CSU-EPSA, Vol. 16, No. 6, 2004, pp. 5-18, 23

[34] X. S. Zhang, S. X. Zhang and Y. Yuan, "Grid Planning for Distribution Network with Distributed Wind Generators," Power System Protection and Control, Vol. 40,No. 13, 2012, pp. 1-6.

[35] R. Billinton, Bagen and Y. Cui, "Reliability Evaluation of Small Stand-alone Wind Energy Conversion Systems Using a Time Series Simulation Model," IEE Proceedings-Generation, Transmission and Distribution, Vol. 150, No. 1, 2003, pp. 96-100.doi:10.1049/ip-gtd:20030068

[36] Bagen and R. Billinton, "Evaluation of Different Operating Strategies in Small Stand-Alone Power Systems," IEEE Transactions on Energy Conversion,Vol. 20, No. 3, 2005, pp. 654-660. doi:10.1109/TEC.2005.847996

[37] C. Q. Liu and Y. Zhang, "Distribution Network Reliability Considering Distribution Generation," Automation of Electric Power Systems, Vol. 31, No. 22, 2007, pp. 46-49.

[38] H. M. Wang, "Reliability Evaluation of Distribution System Including Distributed Generations," Ph.D. Thesis, Tianjin University, Tianjin, 2012.

[39] M. H. J. Bollen, Y. Sun and G. W. Ault, "Reliability of Distribution Networks with DER Including Intentional Islanding," International Conference on Future Power Systems, Amsterdam, Holland, November 2005, pp. 1-6.

[40] Y. Cheng, B. X. Zhou, N. Lin and X. Y. Wang, "Islanding Method in Distribution Networks with the Consideration of Load Management," Proceedings of the CSU-EPSA, Vol. 24, No. 3, 2012, pp. 101-106, 115. 